the stomach contents and his condition which was until now, first class, commenced to fail and color became cyanotic. The incision was rapidly closed in layers with chromic gut, cumol gut and silver wire. The stomach was washed out immediately after the operation and a foul-smelling, yellowish-brown, fecal fluid siphoned away. Magnesia-sulphate, 2 oz., was placed in the stomach with the aid of the stomach tube. The administration of stimulants and oxygen had no effect. The dyspnea became more marked, the cyanosis deepened and he died one-half hour after the operation.

His death was due to the entrance of the stomach contents into the lungs, as shown by the autopsy.

\section{PERILOUS CALMS OF APPENDICITIS}

by robert Wallace hardon, m.D., chicago.

THE patient died, who only a few hours before seemed to be doing so well, with a more normal pulse and temperature, with practically no pain, able to move about with comparative ease, and when those about him were led to think he was getting well. The surgeon who saw the case, before death, either refused to operate, finding a practically comatose patient, or operated only to find the results of a perforated appendix or the results of bacterial extension, affecting more or less and to a greater or less extent the abdominal contents, and the general system.

Deaver, in the Journal, 1904, page 860, says: " Every physician has had one case of severe acute appendicitis, which may have caused difficulty in diagnosis, has referred the patient to a surgeon, stood beside the operating table and observed a highly-inflamed appendix, perhaps gangrenous, removed. And yet this same physician a few months or years later is called to see another patient in the throes of appendiceal colic, knows well what the disease may lead to, and yet gazes, fascinated as if by a rattlesnake, temporizes and dallies until the right iliac fossa becomes ripe and filled with pus."

Why was not the patient operated upon before, at a time when operative results by skilful operators show practically no deaths? First, because of the large percentage of recoveries without operation. Second, because the attending physisian or the family or patient hope that this case will be one of a large majority of recoveries without operation. Third, after waiting some hours, or a few days, the patient is apparently improving, and recovery is taking place, with apparently more or less subsidence of the active inflammatory process. This apparent subsidence of symptoms, the more normal pulse and temperature, the lessened pain may be, and frequently is, the precursor of symptoms and conditions much more grave and menacing to life than the more active ones. It often is the treacherous calm to be followed by the death storm, if prompt action is not taken or allowed.

A few examples will serve to illustrate the subject before going further:

* Read at the Surgical Section of the Mississippi Valley Medical Society, Cincinnati, October, 1904.
CASE I. F. H., forty-four, male; saloon keeper. Seen Nov. 25, 1903, at noon. A large consumer of whiskey, but never drunk, using about a quart of whiskey daily for many years. Patient obese. Temperature $100^{\circ}$ F; pulse 96. Arteries hard. Examination of abdomen revealed some pain on pressure, covering practically the entire region below umbilicus on right side. This area was also somewhat dull on percussion.

Ten days previous there was general abdominal pain, supposed to be neuralgic in character. For this an alkaline cathartic was given. Two days following the beginning of the attack, the pain became localized in the right inguinal region, but was much less intense than at first, and remained in this region up to the time when I first saw the patient.

No history of chills could be elicited. He was told that he had appendicitis, which had extended to the abdominal cavity, causing peritonitis with pus, and advised to go to the hospital at once and submit to operation. Refusing, he was advised to lie perfectly quiet, given a low enema, and all food and fluid by mouth cut off. Two days later he consented to go to the hospital, the treatment having given him no relief. On entrance at 5 P.M., his pulse was 96 ; temperature $101.8^{\circ}$; respirations 28 . Operation was refused at that time. He was kept quiet and given enemata of normal salt solution, one pint every four hours, if awake and nothing was allowed by mouth. The urine showed $1 \%$ of albumin, mixed hyaline and granular casts. The blood showed a marked leucocytosis, 29,000 being reported.

The second day in the hospital he had a chill at 2 A.M.; temperature of $104.4^{\circ}$; pulse 128. Temperature at 8 A.M. next day was $100.6^{\circ}$; pulse 100 . At noon, temperature $99.4^{\circ}$; pulse 104 . At 8 P.M., temperature $100.4^{\circ}$; pulse 90 . The following day, after having been seen by two of my colleagues at the hospital, he was operated upon, it being considered his only chance. He died. The operation revealed a general peritonitis, with some slight adhesions in the right lower abdomen, holding over a quart of thick, foul pus, having a fecal odor; a sloughed appendix, with only the stump remaining. The treacherous calm had passed; the storm was on, and the wreck came.

CaSE II. Miss D. S., twenty-six. This patient had been under my care at times for some years. Her past history as concerns this attack was of pain two years ago in the right lower abdominal region, thought to be ovarian, and for which no medical advice was sought.

On March 26, 1904, she ate some crab salad at a restaurant. The next day she had some discomfort in the right iliac region, and burning of her stomach. This was followed in a few hours by colicky pains in the right iliac region.

On March 28, she vomited twice. The family, thinking the trouble one of indigestion from the crab salad, gave her a cathartic, and applied antiphlogistine poultices and hot fomentations, with opium. The patient was first seen March 29, at 4 P.M., having a temperature of $102.8^{\circ}$, and pulse of 120 . There was great rigidity of the muscles about the right iliac region, with pain most marked below the external to McBurney's point.

The diagnosis of appendicitis was made, and removal to the hospital advised. The family, still thinking that there was a possibility of indigestion, asked for and got a consultant. The diagnosis was confirmed, but although the patient had a fairly easily palpable abdomen, neither the consultant, Dr. Alfred C. Croftan, nor myself could feel the appendix, partly because of the muscular rigidity and partly because of its posi- 
tion. She entered the hospital at 9 P.M., with a temperature of $100.2^{\circ}$; pulse 108 ; respirations 24 . She was prepared for operation. On my return to the hospital at 10.30 P.M., the temperature was $100.2^{\circ}$; the pulse had dropped to 88 , and there was no pain except on pressure in the right iliac region, and this was less than when first seen. There was, houever, greater tympany than when examined at nine.

I advised operation at once. The family again referred to the crab salad, and said, "It is only a belly ache; see how much better she is." I insisted upon consultation, believing it to be a perilous calm, and it was granted. The consultant agreed with me that operation was imperative, and it was performed at about 1 A.M. The operation took forty minutes, because of many old, firm adhesions of the proximal third of the appendix, and many new of the distal twothirds. It was a muscle-splitting operation, with skin incision $1 \frac{7}{5}$ inches long. Later on the day of operation the pulse at $10 \mathrm{~A} . \mathrm{M}$. was 88 ; temperature $1.00 .2^{\circ}$; respirations 24 . At 6 P.M., pulse 86 ; temperature $98.4^{\circ}$; respirations 22 . During the day she was given liquids by mouth. The day following she was slipped out of bed to use a commode, the pulse and temperature being normal, and two days following was rested in a chair out of bed. The patient left the hospital nine days after operation, following an uneventful recovery.

This one of two recently removed appendices I have brought here as being of sufficient interest to show. This appendix was placed well down on the internal part of the cecum, having a course first anterior, then upwards, then backwards, and downwards, the curved portion being that held by dense old adhesions.

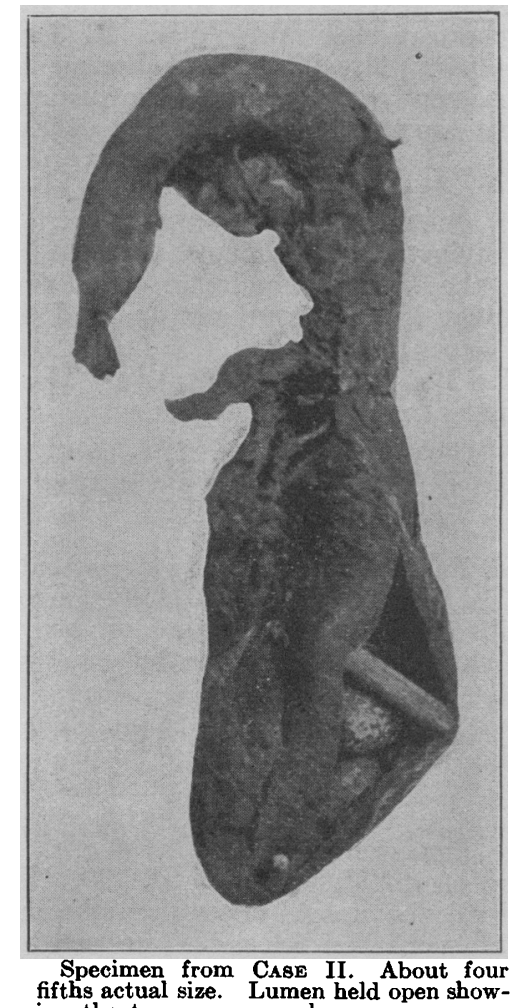
ing the two size. Lumen

Pathological report in part: Length, $5 \frac{1}{2}$ inches. The distal end is greatly enlarged, swollen, and of a dark purplish color, extending two thirds of the way up the organ to a point where a stricture is found almost obliterating the lumen. The capillaries and vessels over the surface are greatly distended. Upon opening into the lumen there were found two full-sized orange seeds. No free pus, but some fecal contents. The pathological histology of sections of this appendix shows extensive round cell infiltration throughout the mucous membrane, and glands almost entirely destroyed, as well as a part of the muscular coat in places. The blood vessels were dilated and congested. There was also an interstitial hyperplasia, showing that a chronic form of disease had existed prior to the last attack.

The picture shows an acute catarrhal condition, with extensive necrosis.

Case III. Mrs. G. W. S., twenty-two years. First seen Aug. 6, 1904, at noon. Temperature $103^{\circ}$; pulse 124 ; respirations 26 . She had marked pain in right iliac region, with rigidity of the muscles on both sides, less in left iliac region. She gave a history of difficult and painful micturition ten days before, with much swelling of the labia, which had subsided under the use of hot douches. Menstruation was normal. There had been no sickness since childhood, but she had not felt well since leaving Arizona in April. The day before, and at 2 A.M. on the day of visit, she had vomited three times, which was attributed to some medicine which had been taken. Vaginal examination showed some discomfort in vagina and tenderness of uterus and adnexa. A diagnosis of appendicitis and infection of uterus and tubes was made, and patient was sent to hospital.

Temperature at entrance at 3 P.M., 103.2; pulse 120; respirations 26 . She was prepared for operation for appendicitis. Temperature at 8 P.M., $101^{\circ}$; pulse 110 ; respirations 24 . Temperature at 11 P.M., $100^{\circ}$; pulse 90 ; respirations 24 .

At this time the patient felt much better, having a less rapid pulse and much lower temperature, but examination revealed increased rigidity of the muscles in the right iliac region, and a particularly painful point below and external to McBurney's point. Vaginal smears showed gonococci. She was operated upon about midnight, and a slightly enlarged congested appendix containing in its distal end one large grape seed was found. The proximal end was somewhat constricted, so that it was barely possible to force through a probe from the distal end. A culture from the lumen showed a pure colon bacillus. An uneventful short recovery followed, she being in the hospital ten days. It is of passing interest to know that she last ate Tokay grapes in Arizona in April; also that her douche bag had been used by others using a common bathroom.

Case IV. Mr. A. M., thirty; married. Fairly developed and nourished. Previous history: About one year previous to present attack was sick in bed for three weeks, with a diagnosis of typhoid fever, although no Widal reaction was found.

First seen, Oct. 17, 1902, at about 7 P.M. Facial expression drawn. Movements caused some pain in right inguinal region. He had been in bed two days; had not vomited, but the pain during the morning of the day seen had been very sharp and colicky. Pulse 103; temperature $102.8^{\circ}$; respirations 24 . Pain on palpation of right inguinal region, while marked, allowed sufficient manipulation, so that the appendix could be felt about one and one-half inches outside and below McBurney's point. A diagnosis of appendicitis was made. The patient was sent to the hospital. Passed a good night, sleeping well. In the morning his pulse was 84 , and temperature $99^{\circ}$, but the face was drawn and tympany more marked than on previous night. He was operated on in the morning, and an erect, highly-injected, congested appendix removed. Near the base, the lumen was entirely constricted, and 
many old adhesions were separated, caused without doubt by the attack of the previous year, then thought to be typhoid. On opening the distended appendix it was found full of a thick, reddish-yellow pus. Cultures showed colon bacillus and staphylococcus.

The night following operation he got out of bed twice during the absence of the nurse to pass urine, and was allowed to get out of bed thereafter. The recovery was uninterrupted.

As briefly as possible, the aim of this paper is to try to reduce the unnecessary mortality due to a hope of recovery without operation. It has been tritely said that so many die of appendicitis because so many get well. Nothing could be more true. The one who gives advice against operation in this treacherous disease must assume a grave responsibility, notwithstanding the patient shows an apparent return to a normal condition, no matter what treatment is used. To say that he has never had a death without operation is only saying that he has been fortunate in not having cases that went on to ulceration, necrosis, perforation, peritonitis and general septicemia. The subsidence of one or more combination of symptoms may not mean recovery, but may mean a far more imminently dangerous condition for the patient. The pulse may return to normal and be of normal volume; the temperature may subside or go below normal. The pain may cease. The dead appendix knows no pain. "After the bowel perforates, all peristalsis rapidly ceases, and the silence of the grave broods over the abdomen." However, the treacherous calm is not a complete one. Something abnormal remains; greater tympany ; accelerated pulse; increased pain; drawn facies; or increased muscular rigidity.

As long as the trouble is confined to the appendix, there is no immediate danger. But no one can tell when the trouble will extend to the peritoneum. There are no sharp lines to be drawn, and it is impossible to say when a peritoneum received its infection. Neither it is necessary for the appendix to be perforated for peritonitis and its sequelæ to occur.

These treacherous calms may come at any time during a few hours or days following the acute attack.

G. Dieulafoy well says: "Traitorous calms of appendicitis are often the cause of death. A temporizing or hesitating physician notices with eagerness the seeming defervescence of the trouble, wishing to put off or avoid surgical intervention, believing it will always be time to operate later, between attacks, but nevertheless there follow terrible accidents against which surgical treatment is of no avail, and the patient dies."

\section{CONCLUSIONS.}

(1) Defervescence of symptoms and apparent better condition of a patient do not always mean recovery, but may be the forerunner of a more dangerous condition.

(2) There being no specific for the disease, no matter what treatment is used, the one who procrastinates should shoulder the responsibility for the death.

(3) When a clear diagnosis is made but one treatment should be advised, that of operation as soon as possible under the conditions, or the golden opportunity may be forever gone.

(4) The physician who does not explain the great dangers of delay and the small comparative danger of operation is doing his patient a serious injustice, which often leads to fatal results.

(5) Operation at the proper time usually greatly shortens convalescence, and eliminates all danger from this cause hereafter.

(6) Procrastination is the greatest cause of surgical deaths, operation often being performed as a last resort, when but little hope of recovery exists.

\section{ghedical Bragregis.}

\section{REPORT ON OBSTETRICS.}

BY FRANK A. HIGgINS, M. D., BOSTON.

THE IMPLANTATION OF THE HUMAN OVUM IN THE UTERUS.

MinoT ${ }^{1}$ regards the discovery of the fact that certain tissues can attack and destroy others as one of the most important discoveries of recent years. He states that it is known that certain young tissues of the embryo are in part endowed with a capacity to destroy the tissues with which they come in contact. A tissue appears to produce a destructive agent, presumably chemical in character, from which itself does not suffer under normal conditions, but which will destroy other tissues with which it comes in contact. Present knowledge leads to the belief that such a phenomenon occurs in the early stages of the human ovum, and affords a fairly correct conception of the implantation of ova upon the wall of the uterus.

The ectodermal cells of the chorion increase in size and numbers by proliferation, and wherever they come in contact with the walls of the uterus destructive changes take place in the uterine tissue. To these ectodermal cells he applies the special name, trophoderm. Dr. Minot affirms that it seems scarcely too much to say positively, in the light of our present knowledge that the function of these chorionic cells, the trophoderm, as the means of implanting the human ovum is demonstrated.

Observations indicate that the ovum becomes completely imbedded in the uterine mucous tissue and that the thickness of the mucous membrane at the time of implantation is a little greater than the total diameter of the ovum. The tissue of the uterus which lies adjacent to the ovum preserves its normal structure and shows no change. The idea is that the cells of the ovum dissolve or digest out and absorb the uterine tissue, making a place for the ovum which grows there, at the mother's expense.

\footnotetext{
1 Gynecological Transactions, 1904.
} 